\title{
A Monograph on the Use of Occlusal Identification Media
}

\section{Gopi Chander N}

SRM Dental College, SRM University, Ramapuram, Chennai, India

\begin{abstract}
Anomalous occlusion modifications in prosthesis and restorations cause high spots and produces destructive changes to craniomandibular system. Over the years the occlusal interferences in restorations and rehabilitation procedures have been extensively studied using articulating papers. The lack of understanding in this fundamental and important procedure of Gnathology and continuous evolution in the science of articulator papers presents a major challenge to dentist.
\end{abstract}

Keywords: Articulating paper; Occlusal test material; Occlusion markers; Occlusion indicators

\section{Review}

Anomalous occlusal contacts, or "high spots" in prostheses and teeth restorations result in destructive changes within the craniomandibular system [1,2]. Traditionally, occlusal interferences in restorations and oral rehabilitation procedures have been disclosed using various articulating media commonly known as "articulating paper." The lack of understanding in this fundamental and important procedure within the field of gnathology, along with the continuous evolution in the science of articulating media present a major challenge to dentists [3-5].

Articulating media, variedly called articulating paper, occlusion test materials, occlusion indicators, occlusion markers are available in a range of choices from paper, ribbon, foils, silks, lacquer, sprays, indicating waxes, and of late, computer analyzing systems have been added to the mix. It is available in diverse forms, such as strips, rolls and sheets and can differ in shape - straight, curved and arch form - with commonly available colors such as blue, red and black. The dimensions of the paper are available in microns and classified as thick, thin, extra thin and ultra-thin. The foils produce less smudging and are available in contrasting colors of white and yellow for use with restorations and metal crowns. Each material has its advantages, disadvantages and limitations in their use. An understanding of the science of these materials will aid clinicians in their appropriate usage in varying situations [6].

Articulating paper can be used to first detect occlusal discrepancies in providing justification for directing and implementing corrective treatment procedures. The remedial procedures can then be continued through either Jankelson's active method or by Schular's passive jaw movement method. The contacts can be differentiated as heavy or light pressure contacts. Thereafter, occlusal interferences can be identified with a "two paper" technique. In the first step, a 200 or $100 \mu \mathrm{m}$ identifier paper media is used to evaluate contacts. Following this, a smaller gauge paper is used to identify halos or occlusal interferences. It is relatively simple to identify and correct discrepancies when contrasting colors are used [7-9].

The occlusal test materials previously used served the purpose and had all the desired requisites of good adaptation to teeth, decreased smudging and marking accuracy in both dry and moist conditions on both teeth and restorative surfaces. There did exist, however, a greater limitation with this conventional technique where quantifying of occlusal contacts cannot be accurately assessed [10-12]. Occlusion scans are now becoming more useful in obtaining the same identifications. The digital analyzer, acting as a force gauge, identifies the contacts, measures the contact force, pressures and timing sequence. It assists the clinician in occlusion adjustments of prostheses, restorations and natural teeth. Though this media is regarded as superior, it is expensive and has a learning curve that requires specialist training $[13,14]$. The conventional technique offers a range of thickness of papers, whereas reservations exist in the usage of computer analyzing systems used by some clinicians based on the thickness of their sensors and sterile pouches that may skew the data.

In summary, diverse choices are available in occlusal identification media employed in the evaluation of anomalous occlusal contacts such as thickness, color, material and now, new computer technology; therefore the clinician's understanding of its advantages and limitations becomes more and more essential in the development of healthy, functional chewing systems.

\section{References}

1. Okeson JP (1995) Occlusion and functional disorders of the masticatory system. Dent Clin North Am39: 285-300.

2. Kikuchi M, Korioth TW, Hannam AG (1997)The association among occlusa contacts, clenching effort, and bite force distribution in man. J Dent Res $76: 1316-1325$

3. Millstein PL (1983)An evaluation of occlusal contact marking indicators:a descriptive, qualitative method. Quintessence Int14: 813-836.

4. Turp JC, Greene CS, Strub JR (2008) Dental occlusion: a critical reflection on past, present and future concepts. J Oral Rehabil 35: 446-453.

5. Millstein P, Maya A (2001)An evaluation of occlusal contact marking indicators A descriptive quantitative method. J Am Dent Assoc 132:1280-1286.

6. Millstein P (2008) Know your indicator. J Mass Dent Soc56: 30-31.

7. Gazit E, Fitzig S, Lieberman MA (1986) Reproducibility of occlusal marking techniques. J Prosthet Dent55: 505-509.

8. Kerstein RB (2008) Articulating paper mark misconceptions and computerized occlusal analysis technology. Dent Implantol Update19: 41-46.

9. Kimmel SS (2009) Rationale and technique for achieving occlusal harmony. $N$ Y State Dent J 75:39-43.

10. Carey JP, Craig M, Kerstein RB, Radke J (2007) Determining a relationship between applied occlusal load and articulating paper mark area. Open Dent J1: $1-7$.

*Corresponding author: Gopi Chander N, SRM Dental College, SRM University, Ramapuram, Chennai -89, India, Tel: +91 98407 49441; E-mail: drgopichander@gmail.com

Received December 20, 2013; Accepted February 04, 2014; Published February 06, 2014

Citation: Gopi Chander N (2014) A Monograph on the Use of Occlusal Identification Media. Dentistry 4: 206. doi:10.4172/2161-1122.1000206

Copyright: (c) 2014 Gopi Chander N. This is an open-access article distributed under the terms of the Creative Commons Attribution License, which permits unrestricted use, distribution, and reproduction in any medium, provided the original author and source are credited. 
11. Qadeer S, Kerstein R, Kim RJ, Huh JB, Shin SW (2012) Relationship between articulation paper mark size and percentage of force measured with computerized occlusal analysis. J Adv Prosthodont 4: 7-12.

12. Reiber T, Fuhr K, Hartmann H, Leicher D (1989) [Recording pattern of Occlusal indicators. I. Influence of indicator thickness, pressure, and surface morphology]. Dtsch Zahnarztl Z 44: 90-93.
13. Kalachev YS, Michailov TA, lordanov PI (2001) Study of occlusal-articulation relationships with the help of T-SCAN apparatus. Folia Med (Plovdiv) 43: 8891.

14. Hsu ML, Palla S, Gallo LM (1992) Sensitivity and reliability of the T-Scan system for occlusal analysis. J Craniomandib Disord Facial Oral Pain 6: 1723. 